\title{
Investigation of high-speed combustible gas ignited by a hot gas jet produced in the shock tube
}

Received: 5 November 2004 / Accepted: 20 January 2006 / Published online: 6 May 2006

(C) Springer-Verlag 2006

\begin{abstract}
The high-speed combustible gas ignited by a hot gas jet, which is induced by shock focusing, was experimentally investigated. By use of the separation mode of shock tube, the test section of a single shock tube is split into two parts, which provide the high-speed flow of combustible gas and pilot flame of hot gas jet, respectively. In the interface of two parts of test sections the flame of jet was formed and spread to the high-speed combustible gas. Two kinds of the ignitions, 3-D "line-flame ignition" and 2-D "plane-flame ignition", were investigated. In the condition of 3-D "lineflame ignition" of combustion, thicker hot gas jet than pure air jet, was observed in schlieren photos. In the condition of 2-D "plane-flame ignition" of combustion, the delay time of ignition and the angle of flame front in schlieren photos were measured, from which the velocity of flame propagation in the high-speed combustible gas is estimated in the range of $30-90 \mathrm{~m} / \mathrm{s}$ and the delay time of ignition is estimated in the range of $0.12-0.29 \mathrm{~ms}$.
\end{abstract}

Keywords Ignition · Hydrocarbon fuel · Shock focusing · Separation mode of shock tube

PACS 47.40.Nm; 82.40.Fp

Part of this paper was presented at the 5th International Workshop on Shock/Vortex Interaction, Kaohsiung, October 27-31, 2003.

Communicated by Z. Jiang

C. Wang $(\varangle) \cdot$ Z. Y. Han

Department of Mechanics and Mechanical Engineering of USTC, P.O. Box 4, 230027 Hefei, China

E-mail:wangchun@imech.ac.cn

M. Situ

The 31 st Institute of CASIC, $1^{\#}$, P.O. Box 7208, 100074 Beijing, China

Present address:

C. Wang

Key Laboratory of High Temperature Gas Dynamics (LHD),

Institute of Mechanics of China Academy of Sciences,

No. 15 Beisihuanxi Road, Beijing 100080, China

\section{Introduction}

Flame propagation in the high-speed combustible gas was an important phenomena. The flame propagation in the supersonic flow of combustible gas was a deep background in the applications of the supersonic combustion and the detonation, especially. Various experimental configurations in conventional wind tunnel were used to investigate the performance of flame propagation in the supersonic flow of combustible gas. Comparatively, seldom work on such phenomena in pulsed wind tunnel was conducted. This is due to the short duration time in pulsed wind tunnel. However, there are some special merits in the tests of pulsed wind tunnel. For example, the temperature of the components of test section would not be raised high. Although the optical measurement methods through glass windows could be used to visualize the flowfield, yet it was expensive for a conventional wind tunnel. The flame propagation speed in supersonic gas flow was investigated in a combination facilities of shock tube and shock tunnel [1-3]. That was an effective method for studying flame propagation in supersonic flow, but the synchronization of two shock tubes makes the operation of shock tubes complex. In the present work, a simple new method of single shock tube was established to investigate the phenomena of flame propagation in the high-speed flow of combustible gas.

The shock focusing, acting as an ignition method to ignite the combustible gas premixed, has attracted much attention of researchers in recent years, such as the work done by Borisov et al. [4-9]. Compared to other ignition method, the ignition by the shock focusing has particular advantage, which is potential in some special situation. Chan et al. discussed the effect of shock focusing on the onset of detonation of combustible mixture gas [10]. Up to now, most of the research works on ignition using the shock focusing were concentrated upon the ignition of quiescent for the combustible gas premixed. In this paper, the shock focusing was expected to generate a hot gas jet and to ignite the highspeed combustible gas, which was different from the other work. 


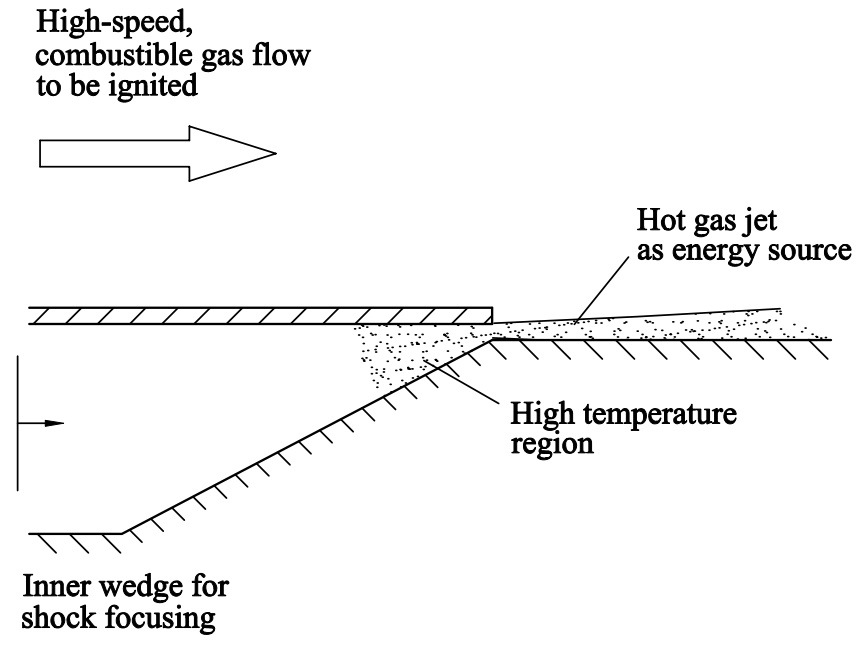

Fig. 1 Shock focusing for the ignition of high-speed flow of the combustible gas

\section{Basic ideas}

For utilizing the shock focusing to ignite the high-speed combustible gas, two conditions were necessary in the experiments: (1) Shock focusing, acting as the energy source, to generate the stable hot gas jet; (2) the hot gas jet, acting as the pilot flame, to ignite the high-speed, combustible gas. Then, the flame propagation from hot gas jet to high-speed combustible gas will be established, as shown in Fig. 1.

It is obvious that two primary test parts are needed. To void the complexity operation of combined facilities of shock tube or shock tunnel, Han has proposed a new operation mode of shock tube, i.e., the separation mode of shock tube, to simulate some phenomena of interaction of complex flows [12]. The separation mode of shock tube is mainly concerned in this paper and used to simulate the interaction of hot gas jet and high-speed mixture gas.

The schematic configuration of separation mode of shock tube is shown in Fig. 2. A shock tube with rectangular cross-sectional area is divided into two parts, upper channel and lower channel. Upper channel is a straight duct, which permits planar shock passing through, and the highspeed flow of combustible gas is induced behind the shock

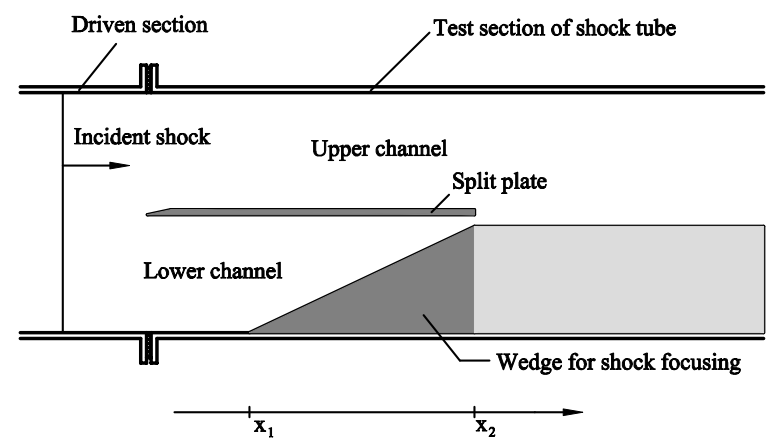

Fig. 2 The schematic configuration of shock tube for separation mode

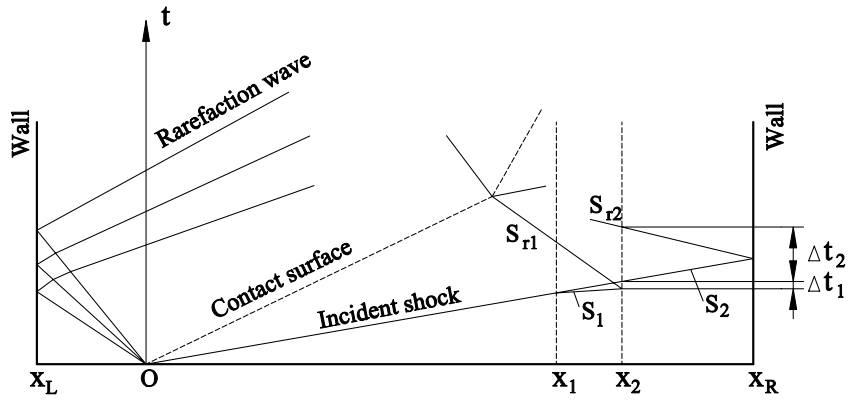

Fig. 3 Schematic operation of separation mode of shock tube

front. In lower channel, a symmetric inner double wedge is inserted to focus the incident shock. At the end of the lower channel, the hole or gap is designed to exhaust the hot gas flow induced by the shock focusing.

When the shock focusing of planar shock in lower channel appears and the temperature and the pressure in the focus are proper, the quiescent combustible gas will be ignited. Then, the combustion products will be exhausted out of the hole or gap. The pilot flame of hot gas jet is formed. In the interface location the flame propagates from the hot gas jet to the high-speed combustible gas. By use of measurement method of schlieren optical system, the flow pattern and the phenomena of flame propagation can be observed.

The schematic operation of separation mode of shock tube is shown in Fig. 3. $x_{1}$ is the place of the start of the shock focusing in lower channel and $x_{2}$ is the end of the lower channel. $S_{1}$ is the focused shock in lower channel. $S_{2}$ is the planar shock in upper channel and $S_{\mathrm{r} 1}$ is the reflected shock in lower channel. The $x_{\mathrm{R}}$ is the right end of shock tube, and the $S_{\mathrm{r} 2}$ is the reflected shock in upper channel.

There are two main test duration times in the whole operation of tests, $\Delta t_{1}$ and $\Delta t_{2} . \Delta t_{1}$ corresponds to the test duration time that shock $S_{1}$ arrives the position of the interface of the upper channel and the lower channel, but $S_{2}$ does not arrive. $\Delta t_{2}$ corresponds to the test duration time that both shock in upper channel and lower channel pass through, and the interaction of hot gas jet and high-speed gas flow takes place until the reflected shock from the right end wall arrives. In our experiments, $\Delta t_{1}$ is less than $10 \mu \mathrm{s}$ and its influence on establishment of flowfield can be neglected. The $\Delta t_{2}$ is the main duration time of shock tube for the separation mode, because the distance between $x_{2}$ and $x_{\mathrm{R}}$ is long enough, about $0.8 \mathrm{~m}$.

\section{Experimental method and facility}

The experiments were conducted in a shock tube with $94 \mathrm{~mm} \times 94 \mathrm{~mm}$ rectangular cross-sectional area. The driver section is 2-m long, driven section is 9-m long and the test section is $1-\mathrm{m}$ long. The configuration near test section is shown in Fig. 4.

Two types of models are used to perform the function of shock focusing, as shown in Fig. 5. In test model 1, the 


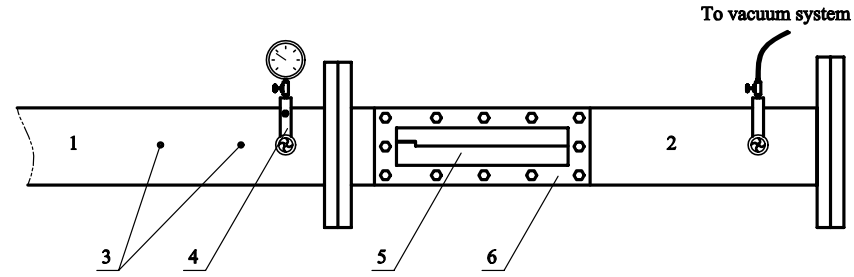

1. Driven section 2. Test section 3. Position of pressure point 4. Position of fuel adding 5. Test model 6. Optical windows

Fig. 4 Schematic configuration near test section

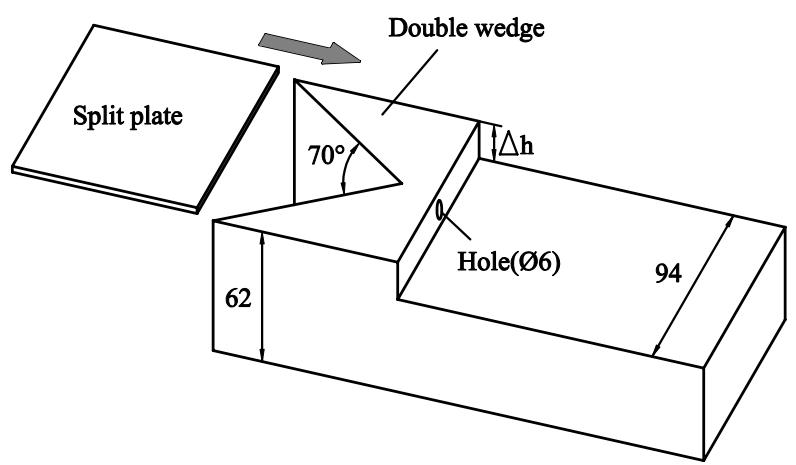

(a) Model 1: 3-D "line-flame ignition" (unit:mm)

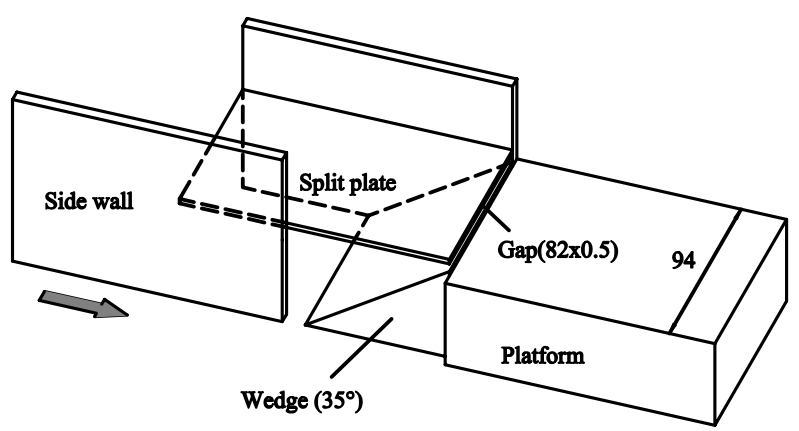

(b) Model 2: 2-D "plane-flame ignition" (unit:mm)

Fig. 5 Two models of shock focusing in lower channel

shock focusing takes place in a $70^{\circ}$ inner double wedge, a 6-mm-diameter hole at the apex of inner double wedges is used to eject the hot gas jet. This model is to realize the 3-D "line-flame ignition" of high-speed combustible gas. In test model 2, the shock focusing takes place in an inner single wedge, the hot gas jet is exhausted out of a gap with $0.5 \mathrm{~mm}$ height and $82 \mathrm{~mm}$ width, which is located on the end of inner single wedge. This model is to realize the 2-D "plane-flame ignition" of the high-speed combustible gas.

When the test starts, the driven section and test section of the shock tube are vacuumed to lower pressure, about $6.6 \mathrm{kPa}$, then a quantity of liquid gasoline is injected into the test section through a valve installed in the test section. After waiting for 5-6 min to insure that the liquid gasoline gets vaporized fully and gets mixed with air well, some fresh air is filled in the test section to form air/gasoline mixture and maintains precalculated fuel/air equivalent ratio and pressure.

In the present tests, the pressure of driver section is about 1.2-1.4 MPa, corresponding to the aluminium diaphragm with $0.4 \mathrm{~mm}$ thickness. By adjusting the pressure of driven section and test section, the various incident shock Mach number can be achieved.

As soon as the aluminium diaphragm between the driver section and driven section is ruptured, an incident shock wave is formed, which travels through the driven section to the test section. After the incident shock wave enters into the test section, it is split into two parts, one moves forwards in the upper channel and maintains its strength. The premixed combustible gas of air/gasoline is heated by the shock wave, but will not be ignited because the temperature behind the shock front is lower than the ignition temperature. The other part of shock wave enters into the cavity of wedge in the lower channel. Because Mach reflection on the surface of the wedge takes place, the shock focusing occurs when the leading shock moves forwards to the apex of the inner wedges. If the temperature behind the shock is high enough, the combustible gas mixed inside the cavity will be ignited by shock focusing. The ignitions of high-speed combustible gas were experimentally investigated and compared for two models, 3-D "line-flame ignition" and 2-D "plane-flame ignition".

\section{Shock focusing and ignition by shock focusing in symmetric, double inner wedge}

The shock focusing in the symmetric inner double wedge was first discussed by Milton [13] in 1989 and was used to ignited the quiescent, combustible mixtured gas by Gelfand [7] in 2000.

When a planar shock enters into a symmetric, inner double wedge, Regular Reflection (RR) or Mach Reflection (MR) on the wall may take place according to different wall angles. It must be noticed that the shock focusing would not take place in case of Regular Reflection on wall, only appears in the condition of Mach Reflection on wall [14].

In case of Mach reflection, when a shock enters into the inner cavity of double wedge, Mach stems on the wall are generated. With the growth of the Mach stems, the triple-points from upper wall and lower wall will be collided. Then the initial incident shock disappears and shock front surface is converted into a non-planar shock which is composed of two Mach stems. Compared with the original shock front, the shock is enhanced, and the temperature and pressure behind the shock front are raised higher. Next, the shock front with two Mach stems cannot move forwards stably. A new Mach stem on the center line will be produced and expanded until its length covers all the cross-sectional area. In the following sequences, these two situations mentioned above will take place alternately and shock will get strengthened step by step. If the space near the apex could be infinitesimal, temperature and pressure just behind the shock front would be raised to infinite theoretically and the 

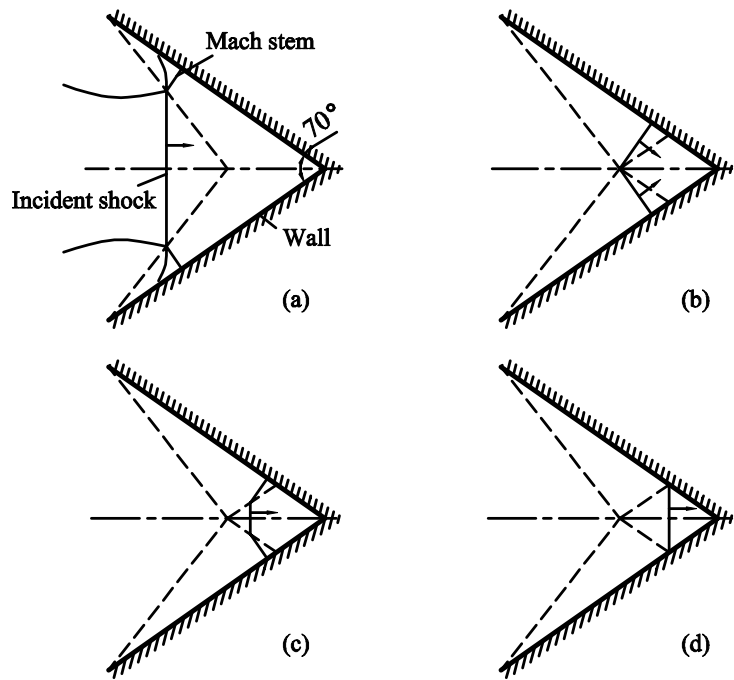

Fig. 6 Shock focusing mechanism in an inner double wedge
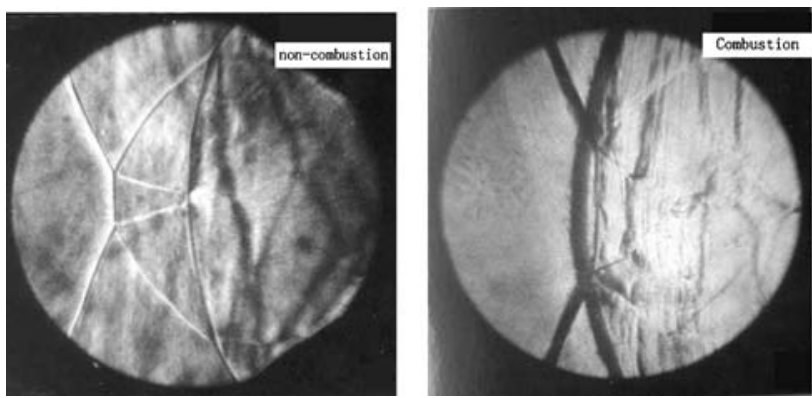

Fig. 7 Schlieren photo of reflected shock in inner wedge in cases of non-combustion and combustion

shock focusing would be established (if the effects of real gas and viscosity are not considered), as illustrated in Fig. 6.

The ignition of quiescent, premixed gasoline/air gas by the shock focusing in symmetric inner double wedge has been studied in precious work [11]. The typical results are shown in Fig. 7. It was compared for the difference of the flowfield of combustion and non-combustion in the condition of shock focusing. The effect of ignition by the shock focusing in inner double wedge was validated. The results show that the onset of deflagration takes place near the apex of symmetric inner double wedge and the reflected shock wave is disturbed.

\section{Results and discussion}

\subsection{Model 1: 3-D "line-flame ignition"}

The test model is shown in Fig. 5a. In this model, the energy source is a hot jet with 3-D "line-flame ignition", which comes from the exit hole of inner double wedge.

Typical schlieren photos are shown in Figs. 8 and 9. The heights of the backward steps, $\Delta h$, are $14 \mathrm{~mm}$ in Fig. 8 and $17 \mathrm{~mm}$ in Fig. 9, respectively. In Figs. 8a and 9a, the shocks

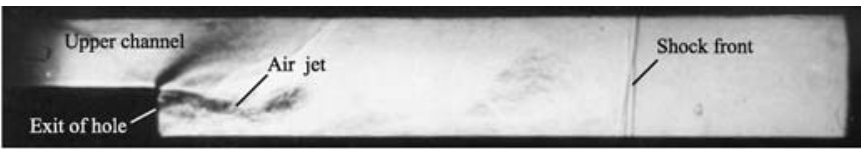

(a) Non-combustion: $M_{s}=1.80, p_{0}=0.0202 \mathrm{MPa}$, $T_{0}=301.2 \mathrm{~K}, \chi=86 \%, t_{\text {delay }}=910 \mu \mathrm{s}$

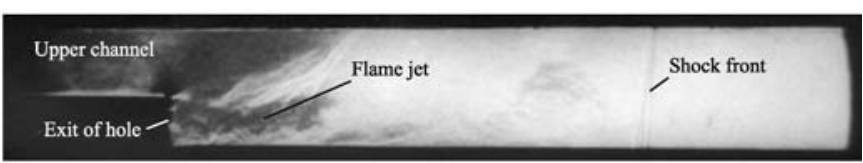

(b) Combustion: $M_{s}=1.80, p_{0}=0.0202 M P a$, $T_{0}=302.2 K, \chi=88 \%, t_{\text {delay }}=910 \mu s(\phi=0.79)$

Fig. 8 Test photos of non-combustion and combustion $(\Delta h=14 \mathrm{~mm})$

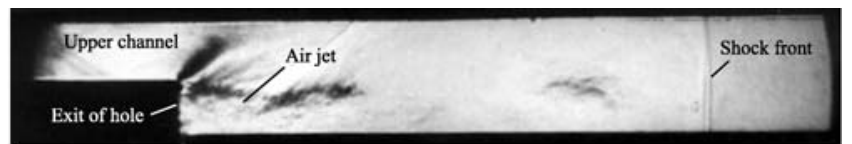

(a) Non-combustion: $M_{s}=1.79, p_{0}=0.0202 M P a$, $T_{0}=303.2 \mathrm{~K}, \chi=57 \%, t_{\text {delay }}=940 \mu \mathrm{s}$

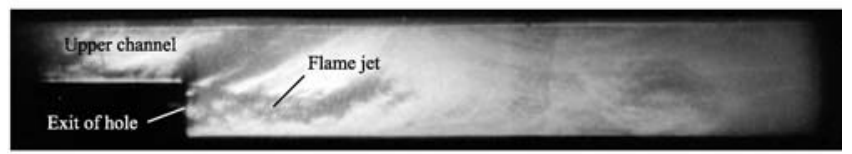

(b) Combustion: $M_{s}=1.80, p_{0}=0.0202 M P a$,

$T_{0}=303.2 \mathrm{~K}, \chi=83 \%, t_{\text {delay }}=960 \mu \mathrm{s}(\phi=0.79)$

Fig. 9 Test photos of non-combustion and combustion $(\Delta h=17 \mathrm{~mm})$

are focused, and the jets out of the hole in the apex of cavity of symmetric inner double wedges were used to heat the air without burning. In Figs. 8b and 9b, the shock is focused on the combustible gas. As the combustible gas is ignited, the hot gas jet is ejected out of the hole in the apex of symmetric inner double wedge, which can be used as pilot flame. The parameters of $p_{0}, T_{0}$, and $\chi$ are initial pressure, temperature, and atmospheric humidity, and $\phi$ is the fuel/gas equivalent ratio.

As shown in Fig. 8a with backward step height $\Delta h=14$ $\mathrm{mm}$, the burning does not appear. The pressure and temperature in the cavity of symmetric inner double wedges are lower, and the jet out of the hole is short and thin. The pressure and temperature are raised high shown in Fig. 8b, because the combustible gas is ignited in the cavity of symmetric inner double wedges. Consequently, the gas jet in condition of combustion displays longer and thicker than that of non-combustion. Besides, due to flame propagation, the jet shown in the Fig. 8b displays thicker than that shown in Fig. 8a.

It must be noticed that the flowfield just behind the leading shock is unsteady and complex, as a shock diffracts around a backward step with the interactions of shock wave and gas jet. If the leading shock moves downwards, the effect of interactions of unsteady waves diminishes gradually.

Same results can be seen in Fig. 9a and b. Clearly, the flame of gas jet out of exit of lower channel is observed in schlieren photos due to flame propagation from the hot gas 


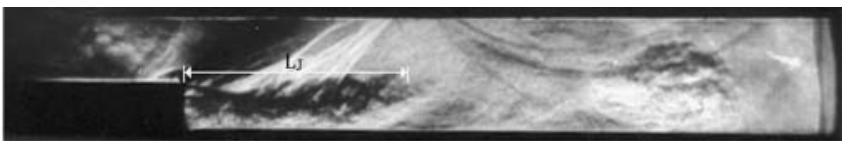

(a) Combustion: $M_{s}=1.79, p_{0}=0.0202 M P a$, $T_{0}=307.2 K, \chi=68 \%, t_{\text {delay }}=960 \mu s(\phi=0.79)$,

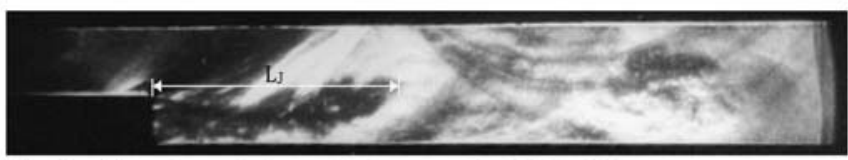

(b) Combustion: $M_{s}=1.82, p_{0}=0.0202 M P a$,

$T_{0}=301.2 K, \chi=64 \%, t_{\text {delay }}=960 \mu s(\phi=1.32)$

Fig. 10 Effect of fuel/air equivalent ratio on jet in case of combustion $(\Delta h=14 \mathrm{~mm})$

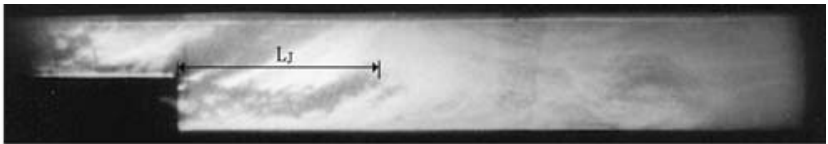

(a) Combustion: $M_{s}=1.80, p_{0}=0.0202 M P a$, $T_{0}=303.2 K, \chi=83 \%, t_{\text {delay }}=960 \mu s(\phi=0.79)$

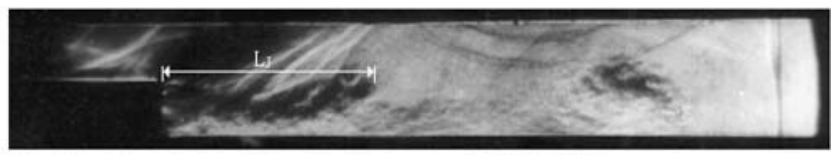

(b) Combustion: $M_{s}=1.79, p_{0}=0.0202 M P a$,

$T_{0}=300.2 K, \chi=67 \%, t_{\text {delay }}=960 \mu s(\phi=1.32)$

Fig. 11 Effect of fuel/air equivalent ratio on jet in case of combustion $(\Delta h=17 \mathrm{~mm})$

jet to high-speed combustible gas, which displays thicker than that of non-combustion. We can also conclude that the position of jet is raised higher when the height of backward step is increased.

The effect of fuel/air equivalent ratio on test results are shown in Fig. $10(\Delta h=14 \mathrm{~mm})$ and Fig. $11(\Delta h=$ $17 \mathrm{~mm})$.

The length of hot gas jet $\left(L_{\mathrm{J}}\right)$ with flame propagation in above tests are shown in Table 1 . The range of $L_{\mathrm{J}}$ is 64 $72 \mathrm{~mm}$.

\subsection{Model 2: 2-D "plane-flame ignition"}

The test model is shown in Fig. 5b. The shock focusingtakes place in an inner single wedge and the hot gas jet is ejected from a gap at the exit of lower channel. In this model, the

Table 1 The length of hot gas jet with flame propagation

\begin{tabular}{lll}
\hline No. & Case (figure) & $L_{\mathrm{J}}(\mathrm{mm})$ \\
\hline 1 & $10 \mathrm{a}$ & 67.7 \\
2 & $10 \mathrm{~b}$ & 71.5 \\
3 & $11 \mathrm{a}$ & 64.8 \\
4 & $11 \mathrm{~b}$ & 67.0 \\
\hline
\end{tabular}

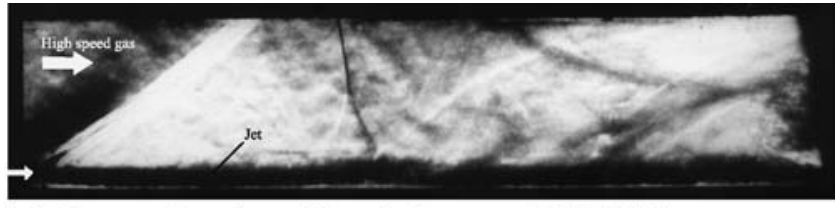

(a) Non-combustion: $M_{s}=1.88, p_{0}=0.0187 \mathrm{MPa}$, $T_{0}=294.2 \mathrm{~K}, \chi=61 \%, t_{\text {delay }}=1000 \mu \mathrm{s}$

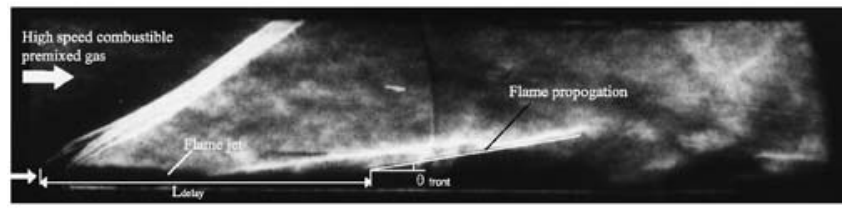

(b) Combustion: $M_{s}=2.02, p_{0}=0.0107 \mathrm{MPa}$,

$T_{0}=294.2 K, \chi=67 \%, t_{\text {delay }}=1000 \mu s,(\phi=1.1)$

Fig. 12 Typical results of non-combustion and combustion in case of "plane-flame ignition"

flame propagation from the hot gas jet to the high-speed combustible gas is a 2-D "plane-flame ignition".

Typical results of non-combustion and combustion are shown in Fig. 12. The parameters $p_{0}, T_{0}$, and $\chi$ are initial pressure, temperature, and atmospheric humidity, $\phi$ is the fuel/gas equivalent ratio. Because the height of backward step is small, the position of hot gas jet out of the exit of lower channel is near the lower wall of test section.

In the non-combustion condition, the gas jet out of the exit of lower channel flows to downstream straightly, although the interactions of gas jet and wave does appear in the flowfield exit shown in Fig. 12a. In the combustion status, i.e., the combustible gas in the inner single wedge is ignited and exhausted from the narrow gap at the exit of lower channel. Thus, the pilot flame is obtained and propagated to the high-speed combustible gas of upper channel with a certain expansion angle after a short distance in downstream direction.

Typically, the mechanism of gas jet flame propagating to the high-speed combustible gas is illustrated in Fig. 13. The high-speed combustible gas is not ignited immediately at the exit of narrow gap. The ignition takes place after a certain ignition delay time. After the ignition, the flame front is produced in the high-speed combustible gas due to the flame propagation. The angle of flame front is usually determined by the velocity of the combustible gas flow and the speed of

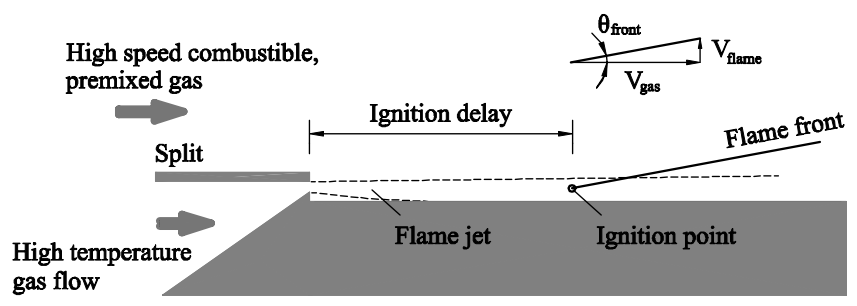

Fig. 13 Mechanism of gas jet flame propagating to the high-speed combustible gas 


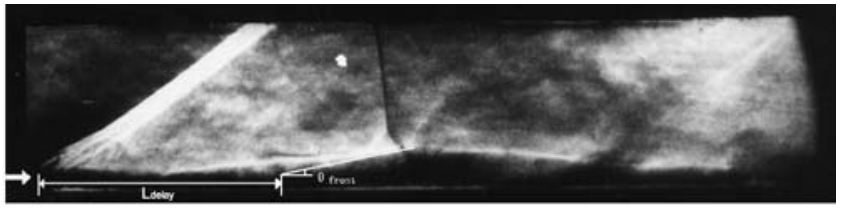

(a) Combustion: $M_{s}=2.00, p_{0}=0.0107 \mathrm{MPa}$, $T_{0}=294.2 K, \chi=67 \%, t_{\text {delay }}=1000 \mu s(\phi=0.79)$

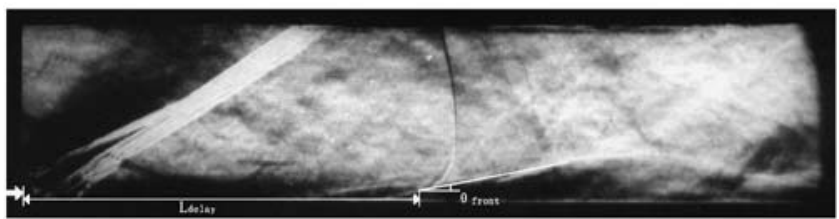

(b) Combustion: $M_{s}=2.10, p_{0}=0.0097 \mathrm{MPa}$,

$T_{0}=295.2 K, \chi=70 \%, t_{\text {delay }}=1000 \mu s(\phi=1.1)$

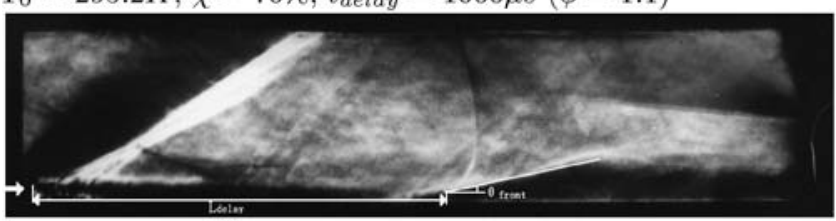

(c) Combustion: $M_{s}=2.16, p_{0}=0.0072 M P a$,

$T_{0}=295.2 \mathrm{~K}, \chi=70 \%, t_{\text {delay }}=1000 \mu s(\phi=1.2)$

Fig. 14 Results of combustion in case of "plane-flame ignition" with different incident shock Mach number

flame propagation in transverse direction, that is,

$\tan \left(\theta_{\text {front }}\right)=\frac{V_{\text {flame }}}{V_{\text {gas }}}$

The test results with different incident shock Mach number are shown in Fig. 14. It can be seen that the ignition position is changed as the Mach number of incident shock is increased.

The data of the delay distance of ignition and the flame propagation angle are shown in Table 2. According to it, the delay time of ignition and the speed of flame propagation in the high-speed combustible gas can be estimated.

Generally, the pressure of the gas in the symmetric inner double wedge is much higher than that in the downstream gas flow, therefore the hot gas jet out of the exit of lower channel is sonic, which is ejected parallel into the supersonic flow in the test section. From the velocity of gas flow in upper channel and the angle of flame propagation, the speed of flame propagation in the high-speed combustible gas is estimated in the range of 30-90 m/s. The delay time of ignition is in the range of $0.12-0.29 \mathrm{~ms}$.

Table 2 The delay distance of ignition and flame propagation angle

\begin{tabular}{llll}
\hline No. & Case (figure) & $L_{\text {delay }}(\mathrm{mm})$ & $\theta_{\text {front }}\left({ }^{\circ}\right)$ \\
\hline 1 & $12 \mathrm{~b}$ & 67.7 & 9.9 \\
2 & $13 \mathrm{a}$ & 71.5 & 12.5 \\
3 & $13 \mathrm{~b}$ & 64.8 & 10.7 \\
4 & $13 \mathrm{c}$ & 67.0 & 13.3 \\
\hline
\end{tabular}

\subsection{Wave structures near backward step}

In the most our tests, the incident shock Mach number is lower than the critical shock Mach number $\left(M_{\mathrm{cr}}\right.$, the shock Mach number which can induce supersonic gas flow behind shock front), and the gas flow behind shock front should be subsonic. But in all schlieren photos of tests, there exist the wave structures near the backward steps.

When a moving shock runs out of the open end of a tube to match the pressure condition, a rarefaction wave will be generated and moving to the upstream of tube. The flow in shock tube will be accelerated by the rarefaction wave. In high subsonic situations, the flow can be accelerated to sonic at the end of tube and to supersonic downstream of the open end of tube. Figure 15 illustrates the basic flow pattern and its $p-u$ diagram.

In this paper, same as the above mechanism illustrated in Fig. 15, unsteady reversed rarefaction waves can be induced, which propagate to upstream and accelerate the gas flow, when a planar shock propagates around the backward step. In the condition of high subsonic flow behind shock front, the gas flow can be accelerated to sonic at the place where channel suddenly expands. After the backward step, the steady expansion wave is obtained to accelerate the gas flow to supersonic. So the downstream flowfield of the backward step is supersonic, which can be validated by the wave structures of the flowfield in the visualized scope. This means that the flame propagation is in the supersonic flow of the combustible gas. The wave structures near the backward step are shown in Fig. 16.

Because the flow is accelerated to supersonic, the special wave structures will be induced in the supersonic flow . In the flowfield, the shock wave will interact with the jet, the shear layer, and the vortex. It should be noted that the optical window does not cover the whole cross-section of the
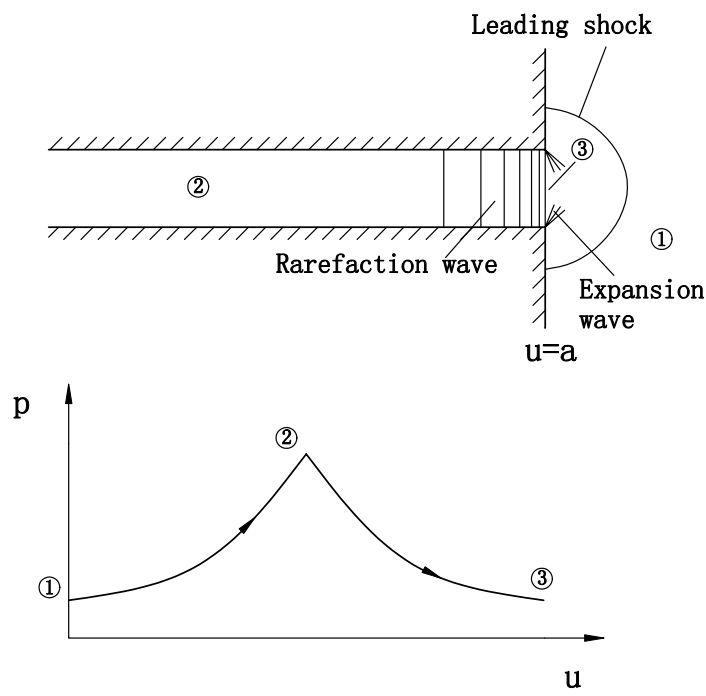

Fig. 15 Flow being accelerated to supersonic while a shock moving out of an open end in condition of high subsonic gas flow behind incident shock 


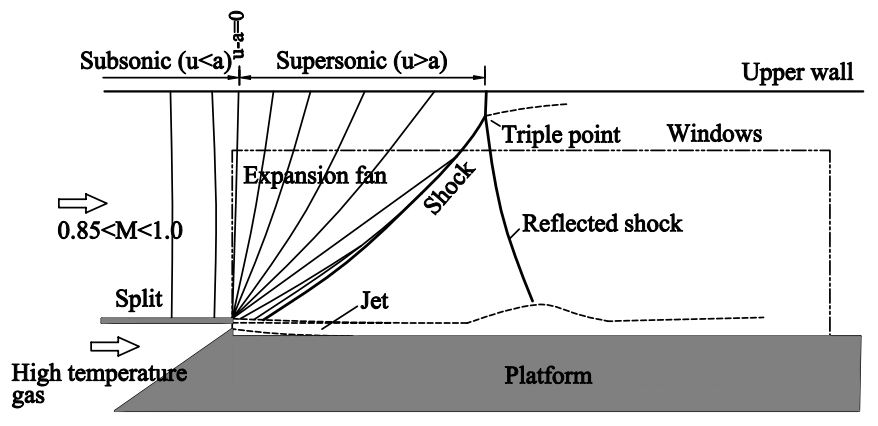

Fig. 16 Wave structure near the backward step in experiment

flowfield. Some part in the upper channel is not shown in the test schlieren photos, the position of windows is also shown in Fig. 16.

\section{Concluding remarks}

1. The separation mode of shock tube in the present work is successfully applied to investigate the phenomena of flame propagating to the high-speed combustible gas. Compared to the other operation modes of shock tubes (shock tunnel), the separation mode has special merits, such as the low cost and simple operation.

2. In the combustion of 3-D "line-flame ignition", the hot gas jet is shown to be thicker and longer than pure air jet, in schlieren photos, which is the evidence of ignition of high-speed combustible gas.

3. In the combustion of 2-D "plane-flame ignition", the delay time of ignition and the angle of flame propagation can be observed. The method of 2-D "plane-flame ignition" is more efficient to investigate the phenomena of flame propagating to the high-speed combustible gas. In the current work, the speed of flame propagation in the high-speed combustible gas is estimated in the range of $30-90 \mathrm{~m} / \mathrm{s}$, and the ignition delay time is in the range of $0.12-0.29 \mathrm{~ms}$.

4. In the condition of high subsonic gas flow after incident shock, the flowfield is accelerated to supersonic by the unsteady rarefaction wave and the steady expansion wave when a planar shock does propagate around the backward step. The flame propagating to supersonic flow of the combustible gas is observed in the experiments.

\section{References}

1. Sun, Y.Y., Situ, M., Han, Z.Y., Luo, X.S., Dong, S.T., Xu, S.L.: New method for the investigation of hydrocarbon-fueled supersonic combustion. Tuijin Jishu/J. Propulsion Tech. 22(1), 69-71 (2001)

2. Han, Z.Y., Sun, Y.Y., Situ, M.: Ignition and flame propagation in a supersonic, combustible flow of hydrocarbon fuel-air mixture. In: Proceedings of the 23rd International Symposium on Shock Wave, Fort worth, p. 7 (2001)

3. Han, Z.Y., Ge, J.B., Yin, X.Z.: An experimental investigation of the influence of streamwise vortex on flame propagation in a supersonic air-hydrocarbon fuel mixture. In: Proceedings of the 4th International Workshop on Shock-Vortex Interaction, p. 10 (2001)

4. Borisov, A.A., Gelfand, B.E., Skatchkov, G.I.: Ignition of gaseous combustible mixtures in focused shock waves. Soviet J. Chem. Phys. 7(12), 1378 (1988)

5. Borisov, A.A., Mikhalkin, V.N., Khomik, S.V.: Experimental studies of detonation propagation in unconfined cylindrical charges of gaseous mixtures. Soviet J. Chem. Phys. 9(6), 1314-1333 (1992)

6. Gelfand, B.E., Frolov, S.M., Medvedev, S.P., Tsyganov, S.A.: Three cases of shock wave focusing in a two-phase combustible medium. In: Proceedings of the 18th International Symposium on Shock Waves and Shock Tubes, pp. 837-842. Sendai, Japan (1991)

7. Gelfand, B.E., Khomik, S.V., Bartenev, A.M., Medvedev, S.P., Groenig, H., Olivier, H.: Detonation and deflagration initiation at the focusing of shock waves in combustible gaseous mixture. Shock Waves 10(3), 197-204 (2000)

8. Bartenev, A.M., Khomik, S.V., Gelfand, B.E., Groenig, H., Olivier, H.: Effect of re-flection type on detonation initiation at shock-wave focusing. Shock Waves, pp. 205-215 (2000)

9. Gelfand, B.E., Khomik, S.V., Medvedev, S.P., Groenig, H., Olivier, H.: Visualization of self-ignition regimes in hydrogen-air mixtures under shock waves focusing. In: Proceedings of the 24th International Congress on High-Speed Photography and Photonics, Sendai, pp. 688-695 (2000)

10. Chan, C.K.: Collision of a shock wave with obstacles in a combustible mixture. Combust. Flame 100, 341-348 (1995)

11. Wang, C., Han, Z.Y., Situ, M.: Experimental investigation on ignition of combustible mixture by shock focusing. Propulsion Tech. 1 (2004) (in Chinese)

12. Han, Z.Y., Wang, C.: Operation modes of shock tubescombination mode and separation mode. In: Proceedings of the 5th International Workshop on Shock/Vortex Interaction, Kaohsiung (2003)

13. Milton, B.E.: The focusing of shock waves in two-dimensional and axi-symmetric ducts, vol. 384. In: Proceedings of the International Workshop on Shock Wave Focusing, pp. 27-57. Sedai, Japan (1989)

14. Wang, C.: Experimental study on ignition of high-speed, combustibe mixed gas flow by shock focusing. Ph.D. thesis, University of Science and Technology of China (2004)
Acknowledgements This project is supported by the National Natural Science Foundation of China (10072061) and the Grant LHD CAS. 